\title{
FURTHER REMARKS ON RIGIDITY OF HÉNON MAPS
}

\author{
RATNA PAL
}

\begin{abstract}
For a Hénon map $H$ in $\mathbb{C}^{2}$, we characterize the polynomial automorphisms of $\mathbb{C}^{2}$ which keep any fixed level set of the Green function of $H$ completely invariant. The interior of any non-zero sublevel set of the Green function of a Hénon map turns out to be a Short $\mathbb{C}^{2}$ and as a consequence of our characterization, it follows that there exists no polynomial automorphism apart from possibly the affine automorphisms which acts as an automorphism on any of these Short $\mathbb{C}^{2}$ 's. Further, we prove that if any two level sets of the Green functions of a pair of Hénon maps coincide, then they almost commute.
\end{abstract}

\section{INTRODUCTION}

\subsection{Background.}

For a polynomial $P$ in the complex plane, the Julia set $J_{P}$ is the complement of the open set in $\mathbb{C}$ where the sequence $\left\{P^{n}\right\}_{n \geq 1}$ form a normal family locally. The dynamical behavior of the points in the Julia set is extremely chaotic and in general the Julia set of a polynomial has a very complicated structure. It is easy to check that if two polynomials $P$ and $Q$ commute, then their Julia sets coincide. Conversely, it follows from the work of Baker-Erëmenko [1] and Beardon 2] that if $J_{P}=J_{Q}$ for two polynomials $P$ and $Q$ of degree greater than or equal to 2, then

$$
P \circ Q=\sigma \circ Q \circ P
$$

where $\sigma(z)=a z+b$ with $|a|=1$ and $\sigma\left(J_{P}\right)=J_{P}$. The Julia sets of two polynomial in the complex plane, of degree greater than or equal to 2 , coincide if and only if they commute upto a rigid motion in the complex plane. Now onwards, this property of Julia sets will be referred as a rigidity property of Julia sets.

Recently, in [7], an analogue of the above-mentioned rigidity phenomenon has been proved for the Hénon maps in $\mathbb{C}^{2}$ which, by the classification theorem of Freidland-Milnor ([12]), is the most important class of automorphisms in $\mathbb{C}^{2}$ from the point of view of dynamics. The class of Hénon maps consists of the polynomial automorphisms of $\mathbb{C}^{2}$ of the form

$$
H=H_{m} \circ H_{m-1} \circ \cdots \circ H_{1}
$$

where

$$
H_{j}(x, y)=\left(y, p_{j}(y)-\delta_{j} x\right)
$$

with $p_{j}$ a polynomial of degree $d_{j} \geq 2$ with highest degree coefficient $c_{j} \in \mathbb{C}$ and $0 \neq \delta_{j} \in \mathbb{C}$. The degree of $H$ is $d=d_{1} d_{2} \ldots d_{m}$. Interested readers can look at [4, [5] and 6] for a detailed study of the dynamics of Hénon maps.

As in the case of polynomials in $\mathbb{C}$, one can give an analogous definition of Julia sets for Hénon maps in $\mathbb{C}^{2}$. For a Hénon map $H$ in $\mathbb{C}^{2}$, the Julia sets $J_{H}^{ \pm}$are defined as the complement of the open sets in $\mathbb{C}^{2}$ where the sequence $\left\{H^{ \pm n}\right\}$ form normal families locally. Here $H^{ \pm n}$ denote the $n$-fold iterates of $H$ and $H^{-1}$, respectively. It turns out that

$$
J_{H}^{ \pm}=\partial K_{H}^{ \pm}
$$

1991 Mathematics Subject Classification. Primary: 32H02 ; Secondary 32H50.

The author is supported by National Post-Doctoral fellowship, SERB, India. 
where

$$
K_{H}^{ \pm}=\left\{(x, y) \in \mathbb{C}^{2}: \text { the sequence }\left(H^{ \pm n}(x, y)\right) \text { is bounded }\right\},
$$

the set of non-escaping points.

Definition 1.1. Let $H$ be an automorphism on $\mathbb{C}^{2}$. A set $S \subseteq \mathbb{C}^{2}$ is called completely invariant under the map $H$ if $H(S)=S$.

Note that $K_{H}^{ \pm}$are completely invariant under $H$. In [7, for a Hénon map $H$, we proved the following rigidity theorem.

Known Theorem 1 (KT1). Let $F$ be an automorphism of $\mathbb{C}^{2}$ which keeps the non-escaping sets $K_{H}^{ \pm}$completely invariant, then $F$ is a polynomial automorphism. If $\operatorname{deg} F \geq 2$, then either $F$ or $F^{-1}$ is a Hénon map. Further, $F$ shares a close relation with $H$, viz.,

$$
F^{ \pm 1} \circ H=C \circ H \circ F^{ \pm 1}
$$

where $C$ a linear map of the form $(x, y) \mapsto\left(\delta_{-} x, \delta_{+} y\right)$ with $\left|\delta_{ \pm}\right|=1$. Also,

$$
\text { either } F^{m}=\sigma H^{n} \text { or } F^{-m}=\sigma H^{n}
$$

for some $m, n \in \mathbb{N}$ and for some affine automorphism $\sigma$ in $\mathbb{C}^{2}$.

The same techniques, which are used to prove the above theorem, gives the following version of rigidity theorem for Julia sets of Hénon maps.

Known Theorem 2 (KT2). Let $H$ and $F$ be two Hénon maps such that their Julia sets coincide, i.e, $J_{H}^{ \pm}=J_{F}^{ \pm}$, then (1.2) and (1.3) hold. Conversely, if $F$ and $H$ be two Hénon maps satisfying

$$
F \circ H=C \circ H \circ F
$$

where $C:(x, y) \mapsto\left(\delta_{-} x, \delta_{+} y\right)$ with $\left|\delta_{ \pm}\right|=1$ and $C\left(K_{H}^{ \pm}\right)=K_{H}^{ \pm}$, then $J_{H}^{ \pm}=J_{F}^{ \pm}$.

The goal of the present article is to improve and extend the rigidity result of Hénon maps obtained in [7].

\subsection{Main Results.}

Let $H$ be a Hénon map. Now if we start with a polynomial automorphism $F$ of $\mathbb{C}^{2}$ such that $F\left(K_{H}^{+}\right)=K_{H}^{+}$, then we can recover the same relations between $H$ and $F$ as in (1.2) and (1.3) (see $(\mathrm{KT} 1)$ ). This shows that the condition $F\left(K_{H}^{-}\right)=K_{H}^{-}$in $(\mathrm{KT} 1)$ is redundant if we start with a polynomial automorphism $F$ in $\mathbb{C}^{2}$. With these words, we present our first theorem.

Theorem 1.2. Let $H$ be a Hénon map in $\mathbb{C}^{2}$ and $F$ be a polynomial automorphism of $\mathbb{C}^{2}$ which keeps $K_{H}^{+}$completely invariant. Then,

(a) if $\operatorname{deg}(F)=1, F$ is of the form

$$
(x, y) \mapsto(a x+f, d y+g)
$$

with $|a|=|d|=1$ and

(b) if $\operatorname{deg} F \geq 2$, then either $F$ or $F^{-1}$ is a Hénon map and accordingly there exist $m, n \in \mathbb{N}^{*}$ such that

$$
\text { either } F^{m}=H^{n} \text { or } F^{-m}=H^{n} \text {. }
$$

Further, there exists $m \in \mathbb{N}^{*}$ such that

$$
\text { either } F^{m} \circ H=H \circ F^{m} \text { or } F^{-m} \circ H=H \circ F^{-m} \text {. }
$$


The Green functions of a Hénon map $H$ is defined as follows:

$$
G_{H}^{ \pm}(x, y):=\lim _{n \rightarrow \infty} \frac{1}{d^{n}} \log ^{+}\left\|H^{ \pm n}(x, y)\right\|
$$

for all $(x, y) \in \mathbb{C}^{2}$. Here $\log ^{+}(x)=\max \{\log x, 0\}$. The functions $G_{H}^{ \pm}$are continuous, plurisubharmonic, non-negative on $\mathbb{C}^{2}$ and pluriharmonic on $\mathbb{C}^{2} \backslash K_{H}^{ \pm}$vanishing precisely on $K_{H}^{ \pm}$.

In case of Theorem 1.1, [7, since we start with an automorphism $F$ which keeps both $K_{H}^{ \pm}$ invariant, a direct analysis of the possible forms of $F$ (using Jung's theorem, 13]) shows that $F$ (or $F^{-1}$ ) must be a Hénon map. Consequently, it follows immediately that $G_{H}^{+}=G_{F}^{+}$(or $\left.G_{H}^{+}=G_{F}^{-}\right)$. But in the present case, since only the invariance of $K_{H}^{+}$is available, a similar analysis using Jung's theorem does not a priori gurantee that $F$ is a Hénon map, rather we get that $F$ (and hence $F^{-1}$ ) is a regular (hence Hénon-type) map (see Section 2 for the definitions of regular maps and Hénon-type maps). Then it requires some work to show that the Green functions of $H$ and $F$ coincide, i.e., $G_{H}^{+}=G_{F}^{+}$or $G_{H}^{+}=G_{F}^{-}$(one can define Green functions of regular and Hénon-type maps in the similar fashion as in the case of Hénon maps and it is discussed in Section 2). Then we use Lamy's theorem ([14]), to show that some iterates of $H$ and $F$ (or $F^{-1}$ ) coincide, i.e., there exist $m, n \in \mathbb{N}$ such that $F^{m}=H^{n}$ (or $F^{-m}=H^{n}$ ) which shows that $F\left(\right.$ or $F^{-1}$ ) is indeed a Hénon map. In fact, in sprit Theorem 1.2 is similar to Theorem 5.4 in [14.

For each $c>0$, let (see [10])

$$
\tilde{\Omega}_{H, c}^{ \pm}=\left\{(x, y) \in \mathbb{C}^{2}: G_{H}^{ \pm}(x, y) \leq c\right\}, K_{H, c}^{ \pm}=\left\{(x, y) \in \mathbb{C}^{2}: G_{H}^{ \pm}(x, y)=c\right\}, \quad J_{H, c}^{ \pm}=\partial K_{H, c}^{ \pm} .
$$

Note that for $c>0$, the set $K_{H, c}^{+}$has empty interior and thus

$$
K_{H, c}^{+}=J_{H, c}^{+} .
$$

Further define

$$
G_{H, c}^{ \pm}(x, y):=\max \left\{G_{H}^{ \pm}(x, y)-c, 0\right\}
$$

for $(x, y) \in \mathbb{C}^{2}$. The functions $G_{H, c}^{ \pm}$are continuous, plurisubharmonic, non-negative on $\mathbb{C}^{2}$ and pluriharmonic on $\mathbb{C}^{2} \backslash J_{H, c}^{ \pm}$vanishing precisely on $\tilde{\Omega}_{H, c}^{ \pm}$. It can be shown that for a Hénon map $H$, the non-escaping sets are the zero level sets of the Green functions $G_{H}^{ \pm}$, i.e.,

$$
K_{H}^{ \pm}=\left\{(x, y) \in \mathbb{C}^{2}: G_{H}^{ \pm}(x, y)=0\right\} .
$$

Clearly, $K_{H, 0}^{ \pm}=K_{H}^{ \pm}$. Theorem 1.2 gives a characterization of automorphisms in $\mathbb{C}^{2}$ in terms of $H$, which keep $K_{H, 0}^{ \pm}$completely invariant. The next theorem shows that in case $c>0$, there exists no automorphism except possibly the affine automorphisms which keeps $K_{H, c}^{ \pm}$completely invariant.

Theorem 1.3. Let $H$ be a Hénon map in $\mathbb{C}^{2}$. If $F$ is a polynomial automorphism in $\mathbb{C}^{2}$ such that

$$
F\left(K_{H, c}^{+}\right)=K_{H, c}^{+}
$$

for some $c>0$, then $F$ is an affine automorphism of the follwing form

$$
(x, y) \mapsto(a x+b y+f, d y+g) .
$$

In [11], Fornæss showed the existence of so called Short $\mathbb{C}^{k}$. A domain $\Omega$ which can be expressed as an increasing union of unit balls (upto biholomorphism) such that Kobayashi metric vanises identically in $\Omega$, but allows a bounded (above) pluri-subharmonic function, is called Short $\mathbb{C}^{k}$. For a Hénon map $H$, it can be shown that the interior of any non-zero sublevel set of the Green function $G_{H}^{+}$, i.e.,

$$
\Omega_{H, c}=\left\{(x, y) \in \mathbb{C}^{2}: G_{H}^{+}(x, y)<c\right\}
$$


is a Short $\mathbb{C}^{2}$, for any $c>0$ (see [1]). Since $\Omega_{H, c}$ is essentially an increasing union of Euclidean balls in $\mathbb{C}^{2}$ whose automorphism group is well-understood, it is an interesting task to understand the automorphism group of $\Omega_{H, c}$. Since any polynomial automorphism $F$ in $\mathbb{C}^{2}$ which acts as an automorphism of $\Omega_{H, c}$ will keep $K_{H, c}^{+}$completely invariant, a simple application of Theorem 1.3 gives the following proposition.

Proposition 1.4. For any $c>0$, there exists no polynomial automorphism of $\mathbb{C}^{2}$ except possibly the affine automorphisms of the form

$$
(x, y) \mapsto(a x+b y+f, d y+g) .
$$

which acts as an automorphism of $\Omega_{H, c}$.

It follows from Theorem 1.1 in [7] that if the zero level sets of Green functions of two Hénon maps (or the Julia sets) coincide, then they almost commute. We prove that the same is true if any two level sets of the Green functions of a pair of Hénon maps coincide.

Theorem 1.5. Let $H_{1}$ and $H_{2}$ be two Hénon maps of degree $d_{H_{1}}$ and $d_{H_{2}}$, respectively such that

$$
J_{H_{1}, c_{1}}^{+}=J_{H_{2}, c_{2}}^{+} \text {and } J_{H_{1}, d_{1}}^{-}=J_{H_{2}, d_{2}}^{-}
$$

for some $c_{1}, c_{2}, d_{1}, d_{2} \geq 0$, then

$$
H_{2} \circ H_{1}=C \circ H_{2} \circ H_{1} .
$$

Here $C(x, y)=\left(\delta_{+} x, \delta_{-} y\right)$ with $\left|\delta_{+}\right|=e^{c\left(d_{H_{1}}-1\right)\left(d_{H_{2}}-1\right)}$ and $\left|\delta_{-}\right|=e^{d\left(d_{H_{1}}-1\right)\left(d_{H_{2}}-1\right)}$ where $c=$ $c_{1}-c_{2}$ and $d=d_{1}-d_{2}$.

Before we start proving our main theorems, we gather a few preparatory stuff in the next section. Proofs of Theorem 1.2, Theorem 1.3 and Theorem 1.5 appear in Section 3, Section 4 and Section 5, respectively.

\section{Preliminaries}

Readers are referred to [4, [5], 6] and [10] for a detailed study of the material in this section.

For $R>0$, let us first define a filtration of $\mathbb{C}^{2}$ as follows:

$$
\begin{aligned}
& V_{R}^{+}=\left\{(x, y) \in \mathbb{C}^{2}:|x|<|y|,|y|>R\right\}, \\
& V_{R}^{-}=\left\{(x, y) \in \mathbb{C}^{2}:|y|<|x|,|x|>R\right\}, \\
& V_{R}=\left\{(x, y) \in \mathbb{C}^{2}:|x|,|y| \leq R\right\} .
\end{aligned}
$$

For a given Hénon map $H$ of degree $d$, there exists $R>0$ such that

$$
\begin{gathered}
H\left(V_{R}^{+}\right) \subset V_{R}^{+}, H\left(V_{R}^{+} \cup V_{R}\right) \subset V_{R}^{+} \cup V_{R}, \\
H^{-1}\left(V_{R}^{-}\right) \subset V_{R}^{-}, H^{-1}\left(V_{R}^{-} \cup V_{R}\right) \subset V_{R}^{-} \cup V_{R}, \\
K_{H}^{ \pm} \subset V_{R} \cup V_{R}^{\mp} \text { and } \mathbb{C}^{2} \backslash K_{H}^{ \pm}=\bigcup_{n=0}^{\infty}\left(H^{\mp n}\right)\left(V_{R}^{ \pm}\right) .
\end{gathered}
$$

Recall that

$$
K_{H}^{ \pm}=\left\{(x, y) \in \mathbb{C}^{2}: \text { the sequence }\left(H^{ \pm n}(x, y)\right) \text { is bounded }\right\} .
$$

As defined in the previous section, the Green functions

$$
G_{H}^{ \pm}(x, y)=\lim _{n \rightarrow \infty} \frac{1}{d^{n}} \log ^{+}\left\|H^{ \pm n}(x, y)\right\|
$$

for $(x, y) \in \mathbb{C}^{2}$. The functions $G_{H}^{ \pm}$are continuous, plurisubharmonic, non-negative on $\mathbb{C}^{2}$ and pluriharmonic on $\mathbb{C}^{2} \backslash K_{H}^{ \pm}$vanishing precisely on $K_{H}^{ \pm}$. By construction, the following functorial property holds:

$$
G_{H}^{ \pm} \circ H=d^{ \pm 1} G_{H}^{ \pm} .
$$


Both $G_{H}^{ \pm}$have logarithmic growth near infinity, i.e., there exists $R>0$ such that

$$
G_{H}^{+}(x, y)=\log ^{+}|y|+O(1)
$$

in $\overline{V_{R}^{+} \cup V_{R}}$, and

$$
G_{H}^{-}(x, y)=\log ^{+}|x|+O(1)
$$

in $\overline{V_{R}^{-} \cup V_{R}}$. Hence

$$
G_{H}^{ \pm}(x, y) \leq \max \left\{\log ^{+}|x|, \log ^{+}|y|\right\}+C
$$

for all $(x, y) \in \mathbb{C}^{2}$ and for some $C>0$. The supports of the positive closed $(1,1)$ currents

$$
\mu_{H}^{ \pm}=d d^{c} G_{H}^{ \pm}
$$

are $J_{H}^{ \pm}$and $\mu_{H}=\mu_{H}^{+} \wedge \mu_{H}^{-}$is an invariant measure for $H$.

Recall that, for each $c>0$, we define (see [10])

$$
\tilde{\Omega}_{H, c}^{ \pm}=\left\{(x, y) \in \mathbb{C}^{2}: G_{H}^{ \pm}(x, y) \leq c\right\}, K_{H, c}^{ \pm}=\left\{(x, y) \in \mathbb{C}^{2}: G_{H}^{ \pm}(x, y)=c\right\}, J_{H, c}^{ \pm}=\partial K_{H, c}^{ \pm}
$$

and recall that $K_{H, c}^{+}=J_{H, c}^{+}$for $c>0$. Further define

$$
G_{H, c}^{ \pm}(x, y)=\max \left\{G_{H}^{ \pm}(x, y)-c, 0\right\}
$$

for $(x, y) \in \mathbb{C}^{2}$. The functions $G_{H, c}^{ \pm}$are continuous, plurisubharmonic, non-negative on $\mathbb{C}^{2}$ and pluriharmonic on $\mathbb{C}^{2} \backslash J_{H, c}^{ \pm}$vanishing precisely on $\tilde{\Omega}_{H, c}^{ \pm}$. Clearly, $G_{H, c}^{ \pm}$satisfy the same inequalities as in (2.1), (2.2) and (2.3). Further, The supports of the positive closed $(1,1)$ currents

$$
\mu_{H, c}^{ \pm}=d d^{c} G_{H, c}^{ \pm}
$$

are $J_{H, c}^{ \pm}$.

The following theorem proved by Dinh-Sibony (see [10]) have been crucially used to establish the main theorems of the present article.

Theorem 2.1. The current $\mu_{H}^{+}$is the unique closed positive $(1,1)$ current of mass 1 supported on $J_{H}^{+}$. For any $c>0$, the current $\mu_{H, c}^{+}$is a closed positive $(1,1)$ current of mass 1 supported on $J_{H, c}^{+}$.

Any Hénon map extends meromorphically to $\mathbb{P}^{2}$ with an isolated indeterminacy point at $I^{+}=$ $[1: 0: 0]$ and similarly, $H^{-1}$ extends to $\mathbb{P}^{2}$ with a lone indeterminacy point at $I^{-}=[0: 1: 0]$. The class of Hénon maps form the most important class of regular maps in $\mathbb{C}^{2}$.

For a polynomial $f$ in $\mathbb{C}^{2}$, let $\hat{f}$ and $\hat{f}^{-1}$ be the meromorphic extensions of $f$ and $f^{-1}$ to $\mathbb{P}^{2}$, respectively. Let $I_{f}^{+}$and $I_{f}^{-}$be the inderminacy points of $\hat{f}$ and $\hat{f}^{-1}$ in $\mathbb{P}^{2}$, respectively.

Definition 2.2. We say that $f$ is regular if $I_{f}^{+} \cap I_{f}^{-}=\emptyset$.

For a regular map $f$ in $\mathbb{C}^{2}$ of degree $d$, the Green functions

$$
G_{f}^{ \pm}(x, y):=\lim _{n \rightarrow \infty} \frac{1}{d^{n}} \log ^{+}\left\|f^{ \pm n}(x, y)\right\|
$$

for $(x, y) \in \mathbb{C}^{2}$. We define

$$
K_{f}^{ \pm}=\left\{(x, y) \in \mathbb{C}^{2} \text { : the sequence }\left(f^{ \pm n}(x, y)\right) \text { is bounded }\right\} \text { and } J_{f}^{ \pm}=\partial K_{f}^{ \pm} .
$$

The functions $G_{f}^{ \pm}$are continuous, plurisubharmonic, non-negative on $\mathbb{C}^{2}$ and pluriharmonic on $\mathbb{C}^{2} \backslash K_{f}^{ \pm}$vanishing precisely on $K_{f}^{ \pm}$. By construction, the following functorial property holds:

$$
G_{f}^{ \pm} \circ f=d^{ \pm 1} G_{f}^{ \pm}
$$


where $d$ is the degree of $f$. Further, the functions $G_{f}^{ \pm}$have logarithmic growth near $I_{f}^{\mp}$ and the similar inequalities as in (2.1), 2.2 and (2.3) hold for $f$. See [18] (Section 2) for the following proposition.

Proposition 2.3. The points $I_{f}^{+}$and $I_{f}^{-}$are the attracting fixed points for $f^{-1}$ and $f$, respectively. Futhermore, for any point $z \in \mathbb{C}^{2} \backslash K_{f}^{ \pm}$,

$$
f^{ \pm n}(z) \rightarrow I_{f}^{\mp}
$$

as $n \rightarrow \infty$.

The supports of the positive closed $(1,1)$ currents

$$
\mu_{f}^{ \pm}=d d^{c} G_{f}^{ \pm}
$$

are $J_{f}^{ \pm}$

The following theorem is due to Dinh-Sibony ([10])

Theorem 2.4. The current $\mu_{f}^{+}$is the unique closed $(1,1)$ current of mass 1 supported on the sets $K_{f}^{+}$and $J_{f}^{+}$.

Definition 2.5. A polynomial automorphism $f$ in $\mathbb{C}^{2}$ is called Hénon-type if

$$
f=\varphi \circ h \circ \varphi^{-1}
$$

where $h$ is composition of Hénon maps and $\varphi$ is a polynomial automorphism in $\mathbb{C}^{2}$.

Clearly, a regular map is a Hénon-type map.

Let $\mathcal{P S H}\left(\mathbb{C}^{2}\right)$ be the collection of all pluri-subharmonic functions in $\mathbb{C}^{2}$. Set

$$
\mathcal{L}=\left\{v \in \mathcal{P S H}\left(\mathbb{C}^{2}\right): v(z) \leq \log ^{+}\|z\|+M \text { with some } M>0\right\}
$$

where $\log ^{+}(x)=\max \{\log x, 0\}$.

Definition 2.6. For a subset $S \subseteq \mathbb{C}^{2}$, the function

$$
L_{S}(z):=\sup \{u(z): u \in \mathcal{L}, u \leq 0 \text { on } S\}
$$

for $z \in \mathbb{C}^{2}$, is called the pluricomplex Green function of $S$.

One can look at Proposition 8.4.10 in [16] for the proof of the following proposition.

Proposition 2.7. The pluricomplex Green functions of the sets $K_{H}^{ \pm}$and of the sets $J_{H}^{ \pm}$are $G_{H}^{ \pm}$.

\section{Proof of Theorem 1.2}

Suppose that $\operatorname{deg}(F) \geq 2$. Then we show that

$F$ is a regular automorphism:

That $F$ is a regular automorphism is obtained following the same line of arguments as in the proof of Theorem 1.1 in [7, which shows that any polynomial automorphism preserving the nonescaping sets $K_{H}^{ \pm}$is essentially a Hénon map. In present case, due to unavilability of invariance of $K_{H}^{-}$under $F$, we need to strech the arguments given in [7] accordingly to conclude that $F$ is a regular polynomial automorphism.

By Jung's theorem (see [13]), $F$ can be written as a composition of affine maps and elementary maps in $\mathbb{C}^{2}$. Recall that an elementary map is of the form

$$
e(x, y)=(\alpha x+p(y), \beta y+\gamma)
$$

where $\alpha \beta \neq 0$ and $p(y)$ is a polynomial in $y$. We consider following cases. 
Case (i): Let

$$
F=a_{1} \circ e_{1} \circ a_{2} \circ e_{2} \circ \cdots \circ a_{k} \circ e_{k}
$$

for some $k \geq 1$ where the $a_{i}$ 's are non-elementary affine maps and the $e_{i}$ 's are non-affine elementary maps. Without loss of generality, suppose that

$$
F=a_{1} \circ e_{1} \circ a_{2} \circ e_{2} .
$$

Let

$$
a_{1}(x, y)=\left(\alpha_{1} x+\beta_{1} y+\delta_{1}, \alpha_{2} x+\beta_{2} y+\delta_{2}\right) .
$$

for $\alpha_{2} \neq 0$. Now consider the maps

$$
a_{1}^{2}(x, y)=\left(\alpha_{2} x+\beta_{2} y+\delta_{2}, s_{2} y+r_{2}\right)
$$

and

$$
a_{1}^{1}(x, y)=(b x+c y, y)
$$

where $b \neq 0, c=\alpha_{1} / \alpha_{2}, r_{2}=\left(\delta_{1}-c \delta_{2}\right) / b$ and $s_{2}=\left(\beta_{1}-c \beta_{2}\right) / b$. Note that $a_{1}=a_{1}^{1} \circ \tau \circ a_{1}^{2}$ where $\tau(x, y)=(y, x)$ for any $b \neq 0$. Expressing $a_{2}$ in a similar way, it follows that

$$
F=a_{1}^{1} \tau a_{1}^{2} e_{1} a_{2}^{1} \tau a_{2}^{2} e_{2} .
$$

Now $a_{1}^{2} e_{1} a_{2}^{1}$ and $a_{2}^{2} e_{2}$ are elementary maps, $E_{1}$ and $E_{2}$ respectively, say. Therefore,

$$
F=a_{1}^{1} \tau E_{1} \tau E_{2}
$$

where $E_{i}(x, y)=\left(m_{i} x+p_{i}(y), k_{i} y+r_{i}\right)$ and the $p_{i}$ 's are polynomials in $y$ of degree at least 2 for $i=1,2$. Since $\tau E_{1}$ and $\tau E_{2}$ are Hénon maps, it follows that $[1: 0: 0]$ is an indeterminacy point of $F$. But $F^{-1}([1: 0: 0])=[1: 0: 0]$. Thus, in this case, $F$ is regular map with $[1: 0: 0]$ as the forward indeterminacy point. Note that the point $[w: 1: 0]$ is the indeterminacy point of $F^{-1}$ for some $w \in \mathbb{C}$.

Case (ii): Let

$$
F=a_{1} \circ e_{1} \circ a_{2} \circ \cdots \circ e_{k-1} \circ a_{k}
$$

for some $k \geq 2$. That $F$ can not be of this form, provided $F\left(K_{H}^{+}\right)=K_{H}^{+}$, follows exactly the same set of arguments as in the proof of Theorem 1.1 in [7] (or case (ii) in Theorem 1.3 in the present paper).

Case (iii): Let

$$
F=e_{1} \circ a_{1} \circ e_{2} \circ a_{2} \circ \cdots \circ e_{k} \circ a_{k}
$$

for some $k \geq 1$. Note that $F^{-1}$ has a form as in Case (i). Since $F^{-1}$ also keeps $K_{H}^{+}$invariant, it follows that $F^{-1}$ is a regular map with $[1: 0: 0]$ as the indeterminacy point. Hence, $F$ is a regular map

Case (iv): Let

$$
F=e_{1} \circ a_{1} \circ e_{2} \circ a_{2} \circ \cdots \circ a_{k-1} \circ e_{k-1} \circ e_{k}
$$

for some $k \geq 1$. For simplicity, we work with

$$
F=e_{1} \circ a_{1} \circ e_{2}
$$

and as in the previous cases, we write

$$
F=e_{1} a_{1}^{1} \tau a_{1}^{2} e_{2}
$$

and thus,

$$
\tau F=\tau e_{1} a_{1}^{1} \tau a_{1}^{2} e_{2} .
$$

Note that both $\tau e_{1} a_{1}^{1}$ and $\tau a_{1}^{2} e_{2}$ are Hénon maps. Thus $\tau F$ is a Hénon map. Therefore, $F[0: 1: 0]=[1: 0: 0]$ and consequently, $F\left(V_{R}^{+}\right)$will intersects $K_{H}^{+}$since $[1: 0: 0]$ is the limit point of $K_{H}^{+}$in $\mathbb{P}^{2}$. This implies, $V_{R}^{+} \cap K_{H}^{+} \neq \emptyset$ since $F\left(K_{H}^{+}\right)=K_{H}^{+}$. This is clearly a contradiction. Therefore, $F$ can not be of this form. 
Thus we prove that $F$ is a regular map. Further, the indeterminacy point of $F$ is either $[1: 0: 0]$ or $[w: 1: 0]$ and accordingly the indeterminacy point of $F^{-1}$ is either $[w: 1: 0]$ or $[1: 0: 0]$.

\section{Green functions of $H$ and $F$ coincide:}

Note that in the previous section, we have shown that if $F\left(K_{H}^{+}\right)=K_{H}^{+}$, then the forms appeared in Case (i) and Case (iii) are the two possible forms of $F$. Now in Case (i), $I_{F}^{+}=[1: 0: 0]$ is the indeterminacy point of $F$. Hence $I_{F}^{+}$is the attracting fixed point for $F^{-1}$ (see Proposition 2.3. Section 2). Now we have $F\left(K_{H}^{+}\right)=K_{H}^{+}$. If $z \notin K_{F}^{+}$, then $F^{n}(z) \rightarrow I_{F}^{-}$as $n \rightarrow \infty$ where $I_{F}^{-}=[w: 1: 0]$ is the indeterminacy point of $F^{-1}$. But $\overline{K_{H}^{+}}=K_{H}^{+} \cup I^{+}$where $I^{+}=[1: 0: 0]$. Therefore, $K_{H}^{+} \subset K_{F}^{+}$. Using Dinh-Sibony rigidity result (see Theorem 2.4, Section 2) for regular maps, we can conclude that

$$
J^{+}=J_{H}^{+}=J_{F}^{+} .
$$

Since $H$ is a Hénon map, $G_{H}^{+}$is the pluricomplex Green function of $J_{H}^{+}$(see 2.7 Section2). Further we claim that $G_{F}^{+}$is the pluricomplex Green function of $J_{F}^{+}$. For $\epsilon>0$ sufficiently small, let

$$
U_{F}^{-}=\left\{(x, y) \in \mathbb{C}^{2}:|x|<(|c|+\epsilon)|y|,|y|>R\right\}
$$

which is clearly away from the point $[1: 0: 0]$. By Theorem 8.4 in [10],

$$
\log \|z\|-M_{2} \leq G_{F}^{+}(z) \leq \log \|z\|+M_{1}
$$

for some $M_{1}, M_{2}>0$ and for all $z \in U_{F}^{-}$. Fix $x_{0} \in \mathbb{C}$ and consider the complex line $L_{x_{0}}=$ $\left\{\left(x_{0}, y\right): y \in \mathbb{C}\right\}$. For $R>0$ sufficiently large

$$
\log \|z\|-K_{2} \leq G_{H}^{+}(z) \leq \log \|z\|+K_{1}
$$

in $V_{R}^{+}$(see (2.1) $)$for some $K_{1}, K_{2}>0$. Now note that

$$
L_{x_{0}}^{R}=\left\{\left(x_{0}, y\right): y \in \mathbb{C},|y|>R\right\} \subseteq U_{F}^{-} \cap V_{R}^{+}
$$

and therefore $G_{H}^{+}-G_{F}^{+}$is bounded at infinity along the line $L_{x_{0}}$. Further, since $J^{+}=J_{H}^{+}=J_{F}^{+}$ and the function $G_{H}^{+}-G_{F}^{+}$is harmonic in $L_{x_{0}} \backslash J^{+}$which vanishes identically on $J^{+}$, it follows that

$$
G_{H}^{+} \leq G_{F}^{+}
$$

Since

$$
G_{F}^{+}(z) \leq \log \|z\|+M
$$

for some $M>0$ and for all $z \in \mathbb{C}^{2}$ and $G_{F}^{+}$vanishes identically on $J_{F}^{+}$,

$$
G_{F}^{+} \leq G_{H}^{+}
$$

in $\mathbb{C}^{2}$. Therefore, the Green function of $H$ and the Green function of $F$ coincide, i.e.,

$$
G_{H}^{+}=G_{F}^{+}
$$

in $\mathbb{C}^{2}$.

In the other case, that is, if $[1: 0: 0]$ is the indeterminacy point of $F^{-1}$, using the similar set of arguments as before, it follows that

$$
G_{H}^{+}=G_{F^{-1}}^{+} .
$$

Some iterates of $F$ and $H$ agree: 
Note that since $F^{ \pm 1}$ are regular maps, they are Hénon-type maps, i.e., $F^{ \pm 1}$ are conjugate to some Hénon maps. Further, without loss of generality, we assume that $G_{H}^{+}=G_{F}^{+}$. Therefore, by Theorem. 5.4 in [14], it follows that there exists $m, n \in \mathbb{N}^{*}$ such that

$$
F^{m}=H^{n} \text {. }
$$

\section{$F$ is a Hénon map:}

Since root of a Hénon map is also a Hénon map (see Theorem. 4.1, [9]), it follows from (3.2) that $F$ is a Hénon map.

$$
\text { Some iterates of } F \text { commutes with } H \text { : }
$$

It follows from (3.2) that

$$
\text { either } F^{m} \circ H=H \circ F^{m} \text { or } F^{-m} \circ H=H \circ F^{-m}
$$

for some $m \in \mathbb{N}^{*}$.

\section{Description of linear automorphisms which keeps $K_{H}^{+}$invariant:}

Let $\sigma$ be an affine automorphism of the form

$$
\sigma(x, y)=(a x+b y+f, c x+d y+g)
$$

such that $\sigma\left(K_{H}^{+}\right)=K_{H}^{+}$. Thus if we take a sequence $\left\{\left(x_{n}, y_{n}\right)\right\}_{n \geq 1} \subseteq K_{H}^{+}$which converges to $[1: 0: 0] \in \mathbb{P}^{2}$, then

$$
\left(a x_{n}+b y_{n}+f, c x_{n}+d y_{n}+g\right) \rightarrow[1: 0: 0]
$$

as $n \rightarrow \infty$, which in turn gives that $c=0$. Hence

$$
\sigma(x, y)=(a x+b y+f, d y+g) .
$$

Now since $\sigma \circ H\left(K_{H}^{+}\right)=K_{H}^{+}$and $\operatorname{deg}(\sigma \circ H) \geq 2$, it follows from previous description that $\sigma \circ H$ is Hénon and thus $b=0$. Therefore

$$
\sigma(x, y)=(a x+f, d y+g) .
$$

Let $(x, y) \in K_{H}^{+}$, then

$$
\sigma^{n}(x, y)=\left(a^{n} x+f \frac{\left(a^{n}-1\right)}{a-1}, d^{n} y+g \frac{\left(d^{n}-1\right)}{(d-1)}\right) .
$$

Now note that if $|a| \leq 1$, then $|d| \leq 1$ since $\sigma^{n}\left(K_{H}^{+}\right)=K_{H}^{+} \subseteq V_{R} \cup V_{R}^{-}$for all $n \geq 1$. Choose $\left(x_{n}, y_{n}\right) \in K_{H}^{+}$such that $\left|x_{n}\right| \rightarrow \infty$ and $y_{n} / x_{n} \rightarrow 0$ as $n \rightarrow \infty$. As in (3.3), we have

$$
\sigma^{n}\left(x_{n}, y_{n}\right)=\left(a^{n} x_{n}+f \frac{\left(a^{n}-1\right)}{a-1}, d^{n} y_{n}+g \frac{\left(d^{n}-1\right)}{(d-1)}\right) .
$$

If $|a|<1$, then it follows from (3.4) that $\sigma^{n}\left(x_{n}, y_{n}\right) \rightarrow[0: 0: 0]$ as $n \rightarrow \infty$ which is a contradiction since $\overline{K_{H}^{+}}=K_{H}^{+} \cup I^{+}$. Thus $|a| \geq 1$. Now

$$
\sigma^{-1}(x, y)=\left(\frac{x}{a}-\frac{f}{a}, \frac{y}{d}-\frac{g}{d}\right) .
$$

Since $\sigma\left(K_{H}^{+}\right)=K_{H}^{+}$, applying same argument as before $\left|a^{-1}\right| \geq 1$. Thus we get $|a|=1$. We have already proved that if $|a| \leq 1$, then $|d| \leq 1$ and thus we have $|d|=1$. So finally we get that

$$
\sigma(x, y)=(a x+f, d y+g)
$$

with $|a|=|d|=1$.

Corollary 3.1. Let $H$ and $F$ be two Hénon maps such that $J_{H}^{+}=J_{F}^{+}$, then there exist $m, n \in \mathbb{N}$ such that $F^{m}=H^{n}$. 


\section{Proof of the Theorem 1.3}

Before starting the proof of 1.3 , we state the following propostion which we shall require later. The proof of the proposition follows exactly as the proof Proposition 2.7, hence we omit the proof.

Proposition 4.1. For any $c>0$, the functions $G_{H, c}^{ \pm}$are the pluricomplex Green functions of the sets $\tilde{\Omega}_{H, c}^{ \pm}$and of the sets $K_{H, c}^{ \pm}=J_{H, c}^{ \pm}$.

Proof of the Theorem 1.3: Let $F$ be a polynomial automorphism of $\mathbb{C}^{2}$ such that $F\left(K_{H, c}^{+}\right)=$ $K_{H, c}^{+}$, for some $c>0$. Then we prove the following equalities:

$$
G_{H, c}^{+} \circ F^{ \pm 1}(x, y)=b^{ \pm} G_{H, c}^{+}(x, y)
$$

for $(x, y) \in \mathbb{C}^{2}$. Note that if (4.1) holds, then $b^{-}=\left(b^{+}\right)^{-1}$ with $b^{+}>0$. The idea of the proof is due to Buzzard-Fornæss ([9]).

Since $F\left(K_{H, c}^{+}\right)=K_{H, c}^{+}$, it follows that $G_{H, c}^{+} \circ F=0$ on $K_{H, c}^{+}$. For any $x \in \mathbb{C}$ and consider

$$
g_{x}(y):=G_{H, c}^{+} \circ F(x, y)
$$

for $y \in \mathbb{C}$. The function $g_{x}$ is harmonic outside the compact set $K_{H, c}^{+} \cap(\{x\} \times \mathbb{C})$ and thus it is harmonic outside a large disk of radius $R>0$. Let $h_{x}$ be the harmonic conjugate of $g_{x}$ in $\{|y|>R\}$ with period $p_{x}$. Therefore

$$
\psi_{x}(y)=g_{x}(y)-p_{x} \log |y|+i h_{x}(y)
$$

is holomorphic in $\{|y|>R\}$. Since

$$
\left|\exp \left(-\psi_{x}(y)\right)\right| \leq|y|^{p_{x}},
$$

the function $\exp \left(-\psi_{x}(y)\right)$ has at most a pole at infinity and thus,

$$
\exp \left(-\psi_{x}(y)\right)=y^{k} \exp f(y)
$$

where $f$ is a holomorphic function in $\{|y|>R\}$ having a removable singularity at infinity. Taking absolute values and then log, we get the following:

$$
g_{x}(y)-p_{x} \log |y|=-k \log |y|-\operatorname{Re}(f(y))
$$

in $\{|y|>R\}$. Therefore,

$$
g_{x}(y)=b_{x} \log |y|+O(1)
$$

in $\{|y|>R\}$ and since $g \geq 0$ in $\mathbb{C}$,

$$
g_{x}(y)=b_{x} \log ^{+}|y|+O(1)
$$

in $\mathbb{C}$.

We prove that $b_{x}$ is independent of $x$. To prove this, we work in a small neighbourhood of a fixed $x_{0}$ and consider $R>0$ large enough. Let $p, q$ be two distinct points near $x_{0}$ and let $I$ be the straight line segment joining them. Then

$$
\Sigma=\{(x, y): x \in I,|y|=R\}
$$

is a smooth real 2-surface with two boundary components namely,

$$
\{(p, y):|y|=R\} \cup\{(q, y):|y|=R\} .
$$

We get

$$
b_{p}-b_{q}=\int_{\partial \Sigma} d^{c}\left(G_{H, c}^{+} \circ F\right)=\int_{\Sigma} d d^{c}\left(G_{H, c}^{+} \circ F\right)=0
$$


applying Stokes' theorem. Here the last equality holds due to the pluriharmonicity of $G_{H, c}^{+} \circ F$ on $V_{R}^{+}$. Thus $b_{x}$ is locally constant and therefore constant everywhere. Let us write $b_{x}=b^{+}$for all $x \in \mathbb{C}$.

Now we have $g_{x_{0}}(y)=b^{+} \log ^{+}|y|+O(1)$ and $G_{H, c}^{+}(x, y)=\log ^{+}|y|+O(1)$ in $V_{R}^{+}$. Further, the difference $g_{x_{0}}(y)-b^{+} G_{H, c}^{+}(y)$ is harmonic at each $y$ for which $\left(x_{0}, y\right) \in \mathbb{C}^{2} \backslash K_{H, c}^{+}$with a removable singularity at infinity and vanishes for $\left(x_{0}, y\right) \in K_{H, c}^{+}$. Therefore, $g_{x_{0}}(y)=b^{+} G_{H, c}^{+}\left(x_{0}, y\right)$ for each $y \in \mathbb{C}$. Applying the same argument we get that $G_{H, c}^{+} \circ F-b^{+} G_{H, c}^{+} \equiv 0$ in $\Delta\left(x_{0} ; r_{0}\right) \times \mathbb{C}$. Since the difference is pluriharmonic in $\mathbb{C}^{2} \backslash K_{H, c}^{+}$and it vanishes in $K_{H, c}^{+}$, we have $G_{H, c}^{+} \circ F=b^{+} G_{H, c}^{+}$ in $\mathbb{C}^{2}$. Using similar arguments we get that

$$
G_{H, c}^{+} \circ F^{-1}=b^{-} G_{H, c}^{+}
$$

in $\mathbb{C}^{2}$ where $b^{-}=\left(b^{+}\right)^{-1}$.

Since for any $c>0, \overline{K_{H, c}^{ \pm}}=K_{H, c}^{ \pm} \cup I^{ \pm}$in $\mathbb{P}^{2}$ (see [10]), if $\operatorname{deg} F=1$, using the similar arguments as in Theorem 1.2, we get that

$$
F(x, y)=(a x+b y+f, d y+g)
$$

for $(x, y) \in \mathbb{C}^{2}$. In case $\operatorname{deg} F \geq 2$, we prove that

$F$ is a regular automorphism:

To prove that $F$ is a regular polynomial automorphism, we shall use the similar set of arguments as in the first part of the proof of Theorem 1.2. As before, the following cases arise:

Case (i): Let

$$
F=a_{1} \circ e_{1} \circ a_{2} \circ e_{2} \circ \cdots \circ a_{k} \circ e_{k}
$$

for some $k \geq 1$ where the $a_{i}$ 's are non-elementary affine maps and the $e_{i}$ 's are non-affine elementary maps. As it is shown in Theorem 1.2, in this case, $F$ is a regular map.

Case (ii) Let

$$
F=a_{1} \circ e_{1} \circ a_{2} \circ \cdots \circ e_{k-1} \circ a_{k}
$$

for some $k \geq 2$. For simplicity, assume that $F=a_{1} \circ e_{1} \circ a_{2}$. As in the previous case we can write

$$
F=a_{1}^{1} \tau a_{1}^{2} e_{1} a_{2}^{1} \tau a_{2}^{2}
$$

where $a_{1}^{1}(x, y)=(b x+c y, y)$ and $\tau a_{2}^{2}(x, y)=\left(s_{2} y+r_{2}, \alpha_{2} x+\beta_{2} y+\delta_{2}\right)$. That $F$ can not be of this form follows exactly as in the proof of Theorem 1.1 in [7]. However, since in our present case $K_{H, c}^{+}$is invariant under $F$ instead of the invariance of the non-escaping set $K_{H}^{+}$(as in Theorem 1.1 in [7]), we need to modify our proof accordingly.

Note that for any given $c>0$, there exists $R_{c}>0$ sufficiently large such that

$$
K_{H, c}^{+} \cap V_{R_{c}}^{+}=\emptyset \text {. }
$$

Let for each $n \in \mathbb{N}$, there exists $\left(x_{n}, y_{n}\right) \in K_{H, c}^{+} \cap V_{n}^{+}$. Now by Lemma 6.3 in [10], $\overline{K_{H, c}^{+}}=$ $K_{H, c} \cup I^{+}$in $\mathbb{P}^{2}$. Therefore $\left[x_{n}: y_{n}: 1\right] \rightarrow[1: 0: 0]$ as $n \rightarrow \infty$ which contradicts the fact that $\left(x_{n}, y_{n}\right) \in V_{n}^{+}$for each $n \geq 1$. Thus (4.2) follows.

Claim: There exists a sequence $\left(x_{n}, y_{n}\right)_{n \geq 1} \subseteq K_{H, c}^{+} \cap V_{R}^{-}$with $\left|x_{n}\right| \geq\left|y_{n}\right| \geq n$ for all $n \geq 1$.

If no such sequence exists, then we can choose a sequence $\left(x_{n}, y_{n}\right) \in K_{H, c}^{+} \cap V_{R}^{-}$such that $\left|y_{n}\right|$ is bounded by a fixed constant $M>1$ for all $n \geq 1$ and $\left|x_{n}\right| \rightarrow \infty$ as $n \rightarrow \infty$. Without loss of generality, we choose $R>0$ sufficiently large such that $K_{H, c}^{+}$and $K_{H, d c}^{+}$both are contained in $V_{R} \cup V_{R}^{-}$where $d=\operatorname{deg} H$. Suppose that the Hénon map is of the form: $H:(x, y) \mapsto$ 
$(y, p(y)-\delta x)$ with $\delta \neq 0$. Then there exists a subsequence $\left\{\left(x_{n_{k}}, y_{n_{k}}\right)\right\} \subset K_{H, c}^{+} \cap V_{R}^{-}$such that $\left\{H\left(x_{n_{k}}, y_{n_{k}}\right)\right\} \subset K_{H, d c}^{+} \cap V_{R}^{-}$and thus

$$
\left|y_{n_{k}}\right| \geq\left|p\left(y_{n_{k}}\right)-\delta x_{n_{k}}\right| \geq|\delta|\left|x_{n_{k}}\right|-\left|p\left(y_{n_{k}}\right)\right| .
$$

Therefore, the sequence $\left\{x_{n_{k}}\right\}$ is bounded which is a contradiction.

Since $\overline{K_{H, c}^{+}}=K_{H, c}^{+} \cup I^{+}$in $\mathbb{P}^{2}$,

$$
\frac{\left|y_{n}\right|}{\left|x_{n}\right|} \rightarrow 0 \text { as } n \rightarrow \infty
$$

Thus

$$
\left|y_{n}\right| \leq \epsilon_{n}\left|x_{n}\right|
$$

for all $n \geq 1$ with $\epsilon_{n} \rightarrow 0$.

Note that $\tau a_{2}^{2}\left(x_{n}, y_{n}\right)=\left(s_{2} y_{n}+r_{2}, \alpha_{2} x_{n}+\beta_{2} y_{n}+\delta_{2}\right)$ and

$$
\begin{aligned}
\left|\alpha_{2} x_{n}+\beta_{2} y_{n}+\delta_{2}\right| & \geq\left(\left|\alpha_{2}\right|-\epsilon_{n}\left|\beta_{2}\right|\right)\left|x_{n}\right|-\left|\delta_{2}\right| \\
& \geq \frac{1}{2}\left|\alpha_{2}\right|\left|x_{n}\right|-\left|\delta_{2}\right| \\
& \geq \frac{1}{2}\left|\alpha_{2}\right|\left|y_{n}\right|-\left|\delta_{2}\right| \geq\left|s_{2}\right|\left|y_{n}\right|+\left|r_{2}\right|\left|y_{n}\right|-\left|\delta_{2}\right|
\end{aligned}
$$

for all $n \geq n_{0}$. Now since $\left|s_{2}\right|$ and $\left|r_{2}\right|$ can be chosen sufficiently small, we choose them such that $\left|\alpha_{2}\right| / 2 \geq\left|s_{2}\right|+\left|r_{2}\right|$. Thus the last inequality follows.

Since $\left|y_{n}\right| \rightarrow \infty$ as $n \rightarrow \infty$, it follows that

$$
\left|\alpha_{2} x_{n}+\beta_{2} y_{n}+\delta_{2}\right| \geq\left|s_{2}\right|\left|y_{n}\right|+\left|r_{2}\right| \geq\left|s_{2} y_{n}+r_{2}\right|
$$

and

$$
\left|\alpha_{2} x_{n}+\beta_{2} y_{n}+\delta_{2}\right| \geq R
$$

for sufficiently large $n$.

Thus for a sequence $\left(x_{n}, y_{n}\right)_{n \geq 1} \subseteq K_{H, c}^{+} \cap V_{R}^{-}$with $\left|x_{n}\right| \geq\left|y_{n}\right| \geq n$, it turns out that $\tau a_{2}^{2}\left(x_{n}, y_{n}\right) \in V_{R}^{+}$for sufficiently large $n$. Thus

$$
\left(x_{n}^{\prime}, y_{n}^{\prime}\right)=\tau a_{1}^{2} e_{1} a_{2}^{1} \tau a_{2}^{2}\left(x_{n}, y_{n}\right) \in V_{R}^{+}
$$

and

$$
\left|b x_{n}^{\prime}+c y_{n}^{\prime}\right| \leq(|b|+|c|)\left|y_{n}^{\prime}\right|
$$

Hence

$$
\left|y_{n}^{\prime \prime}\right| \geq \frac{1}{(|b|+|c|)}\left|x_{n}^{\prime \prime}\right|
$$

where $\left(x_{n}^{\prime \prime}, y_{n}^{\prime \prime}\right)=a_{1}^{1}\left(x_{n}^{\prime}, y_{n}^{\prime}\right)$. Now since $F\left(K_{H, c}^{+}\right)=K_{H, c}^{+}$,

$$
\left(x_{n}^{\prime \prime}, y_{n}^{\prime \prime}\right)=F\left(x_{n}, y_{n}\right) \in K_{H, c}^{+} \cap V_{R}^{-}
$$

for sufficiently large $n \geq 1$ and $\left\|\left(x_{n}^{\prime \prime}, y_{n}^{\prime \prime}\right)\right\| \rightarrow \infty$ as $n \rightarrow \infty$. By (4.3), we get that

$$
\left|y_{n}^{\prime \prime}\right| \leq \epsilon_{n}\left|x_{n}^{\prime \prime}\right|
$$

where $\epsilon_{n} \rightarrow 0$ as $n \rightarrow \infty$ which contradicts (4.4). Thus $F$ cannot be of this form.

Case (iii): Let

$$
F=e_{1} \circ a_{1} \circ e_{2} \circ a_{2} \circ \cdots \circ e_{k} \circ a_{k}
$$


for some $k \geq 1$. Note that $F^{-1}$ has a form as in Case 1 . Since $F^{-1}$ also keeps $K_{H, c}^{+}$invariant, it follows that $F^{-1}$ is a regular map. Hence, $F$ is a regular map with $[w: 1: 0]$ as its indeterminacy point for some $w \in \mathbb{C}$.

Case (iv): Let

$$
F=e_{1} \circ a_{1} \circ e_{2} \circ a_{2} \circ \cdots \circ a_{k-1} \circ e_{k-1} \circ e_{k}
$$

for some $k \geq 1$. For simplicity, we work with

$$
F=e_{1} \circ a_{1} \circ e_{2} .
$$

As in the previous cases, we can write

$$
F=e_{1} a_{1}^{1} \tau a_{1}^{2} e_{2}
$$

and thus,

$$
\tau F=\tau e_{1} a_{1}^{1} \tau a_{1}^{2} e_{2} .
$$

Note that both $\tau e_{1} a_{1}^{1}$ and $\tau a_{1}^{2} e_{2}$ are Hénon maps. Thus $\tau F$ is a Hénon map. Therefore, $F[0: 1: 0]=[1: 0: 0]$ and consequently, $F\left(V_{R}^{+}\right)$will intersects $K_{H, c}^{+}$since $[1: 0: 0]$ is the limit point of $K_{H, c}^{+}$in $\mathbb{P}^{2}$. This implies, $V_{R}^{+} \cap K_{H, c}^{+} \neq \emptyset$ since $F\left(K_{H, c}^{+}\right)=K_{H, c}^{+}$. This is clearly a contradiction. Therefore, $F$ can not be of this form.

Thus we prove that $F$ is a regular map. Furthermore, the point $[1: 0: 0]$ is the indeterminacy point either of $F$ or of $F^{-1}$. Without loss of generality, let $[1: 0: 0]$ be the indeterminacy point of $F$.

$K_{H, c}^{+}$never remains invariant under an automorphism $F$ with $\operatorname{deg} F \geq 2$ and $c>0$ :

From (4.1), it follows that

$$
\max \left\{G_{H}^{+} \circ F(x, y)-c, 0\right\}=b^{+} \max \left\{G_{H}^{+}(x, y)-c, 0\right\}
$$

in $\mathbb{C}^{2}$. Comparing both sides of (4), we get that

$$
G_{H}^{+} \circ F(x, y)=b^{+} G_{H}^{+}(x, y)-b^{+} c+c
$$

for all $(x, y)$ such that $G_{H}^{+}(x, y)>c$. In particular for sufficiently large $R>0$, we have that $G_{H}^{+}(x, y)>c$ for all $(x, y) \in V_{R}^{+}$.

Since

$$
G_{H}^{+} \circ F(z)=b^{+} G_{H}^{+}(z)-b^{+} c+c
$$

for all $(x, y)$ such that $G_{H}^{+}(x, y)>c$, it follows that

$$
F\left(\left\{(x, y) \in \mathbb{C}^{2}: G_{H}^{+}(x, y)>c\right\}\right) \subseteq\left\{(x, y) \in \mathbb{C}^{2}: G_{H}^{+}(x, y)>c\right\} .
$$

Also,

$$
G_{H}^{+} \circ F^{-1}(x, y)=b^{-} G_{H}^{+}(x, y)-b^{-} c+c
$$

for all $(x, y)$ such that $G_{H}^{+}(x, y)>c$, it follows that

$$
F^{-1}\left(\left\{(x, y) \in \mathbb{C}^{2}: G_{H}^{+}(x, y)>c\right\}\right) \subseteq\left\{(x, y) \in \mathbb{C}^{2}: G_{H}^{+}(x, y)>c\right\} .
$$

Therefore,

This implies

$$
F\left(\left\{(x, y) \in \mathbb{C}^{2}: G_{H}^{+}(x, y)>c\right\}\right)=\left\{(x, y) \in \mathbb{C}^{2}: G_{H}^{+}(x, y)>c\right\} .
$$

$$
F\left(\left\{(x, y) \in \mathbb{C}^{2}: G_{H}^{+}(x, y)<c\right\}\right)=\left\{(x, y) \in \mathbb{C}^{2}: G_{H}^{+}(x, y)<c\right\}
$$

since $F\left(K_{H, c}^{+}\right)=K_{H, c}^{+}$.

Claim: $\Omega_{H, c}=\left\{(x, y) \in \mathbb{C}^{2}: G_{H}^{+}(x, y)<c\right\} \subseteq K_{F}^{+}$. 
Since $F\left(K_{H, c}^{+}\right)=K_{H, c}^{+}$, by Prop. 2.3, it follows that $J_{H, c}^{+}=K_{H, c}^{+} \subset K_{F}^{+}$. By rigidity results of Dinh-Sibony (see Theorem 2.1 and Theorem 2.4), it follows that

$$
J_{H, c}^{+}=J_{F}^{+} .
$$

By Prop. 4.1, the function $G_{H, c}^{+}$is the pluricomplex Green function of $J_{H, c}^{+}$. Again as in Theorem 1.2. we can show that $G_{F}^{+}$is the pluricomplex Green function of $J_{F}^{+}$and thus

$$
G_{H, c}^{+}=G_{F}^{+} .
$$

Now since $G_{H, c}^{+}$vanishes identically on $\Omega_{H, c}$, it follows that $G_{F}^{+}$also vanishes identically on $\Omega_{H, c}$. Since

$$
K_{F}^{+}=\left\{(x, y): G_{F}^{+}(x, y)=0\right\}
$$

we have that

$$
\Omega_{H, c} \subseteq K_{F}^{+} .
$$

Now by Theorem 2.1, it follows that for each positive $r<c$, the set $J_{H, r}^{+}$supports a positive closed $(1,1)$ current of mass 1 . On the other hand $K_{F}^{+}$supports a unique positive closed $(1,1)$ current of mass 1 . Contradiction! This finishes the proof.

Thus $F$ must be an affine automorphism. Since $F\left(K_{H, c}^{+}\right)=K_{H, c}^{+}$and $\overline{K_{H, c}^{+}}=K_{H, c}^{+} \cup I^{+}$in $\mathbb{P}^{2}$, applying similar arguments as before, we can show that

$$
F(x, y)=(a x+b y+f, d y+g) .
$$

\section{Proof of the Theorem 1.5}

Let us first state the following propositon (see [7]) which we require to prove Theorem [1.5. Suppose $H$ is of the form (1.1).

Proposition 5.1. For a given Hénon map H, there exist non-vanishing holomorphic functions $\phi_{H}^{ \pm}: V_{R}^{ \pm} \rightarrow \mathbb{C}$ such that

$$
\phi_{H}^{+} \circ H(x, y)=c_{H}\left(\phi_{H}(x, y)\right)^{d}
$$

in $V_{R}^{+}$where

$$
c_{H}=\prod_{j=1}^{m} c_{j}{ }^{d_{j+1} \cdots d_{m}}
$$

with the convention that $d_{j+1} \cdots d_{m}=1$ when $j=m, d=d_{j} \cdots d_{1}$ and

$$
\phi_{H}^{-} \circ H^{-1}(x, y)=c_{H}^{\prime}\left(\phi_{H}^{-}(x, y)\right)^{d}
$$

in $V_{R}^{-}$where

$$
c_{H}^{\prime}=\prod_{j=1}^{m}\left(c_{j} \delta_{j}^{-1}\right)^{d_{j-1} \cdots d_{1}}
$$

with the convention that $d_{j-1} \cdots d_{1}=1$ when $j=1$. Further,

$$
\phi_{H}^{+}(x, y) \sim y \text { as }\|(x, y)\| \rightarrow \infty \text { in } V_{R}^{+}
$$

and

$$
\phi_{H}^{-}(x, y) \sim x \text { as }\|(x, y)\| \rightarrow \infty \text { in } V_{R}^{-} .
$$

The functions $\phi_{H}^{ \pm}$are called the Böttcher functions corresponding to the Hénon map $H$. 
Proof of the Theorem 1.5: Since $K_{H_{1}, c_{1}}^{+}=K_{H_{2}, c_{2}}^{+}$, by Prop. 4.1, we have $G_{H_{1}, c_{1}}^{+}=G_{H_{2}, c_{2}}^{+}$in $\mathbb{C}^{2}$, i.e.,

$$
\max \left\{G_{H_{1}}^{+}-c_{1}, 0\right\}=\max \left\{G_{H_{2}}^{+}-c_{2}, 0\right\}
$$

in $\mathbb{C}^{2}$. Therefore,

$$
G_{H_{1}}^{+}-c_{1}=G_{H_{2}}^{+}-c_{2}
$$

in $V_{R}^{+}$, for $R>0$ sufficiently large. Thus by Prop. 5.1, it follows that

$$
\log \left|\phi_{H_{1}}^{+}\right|+\frac{1}{d_{H_{1}}-1} \log \left|c_{H_{1}}\right|=\log \left|\phi_{H_{2}}^{+}\right|+\frac{1}{d_{H_{2}}-1} \log \left|c_{H_{2}}\right|+c
$$

with $c=c_{1}-c_{2}$ in $V_{R}^{+}$. Also since

$$
\phi_{H_{i}}(x, y) \sim y \text { as }\|(x, y)\| \rightarrow \infty
$$

for $i=1,2$, we have

$$
\frac{1}{d_{H_{1}}-1} \log \left|c_{H_{1}}\right|=\frac{1}{d_{H_{2}}-1} \log \left|c_{H_{2}}\right|+c
$$

which in turn gives

$$
\log \left|\phi_{H_{1}}^{+}\right|=\log \left|\phi_{H_{2}}^{+}\right|
$$

in $V_{R}^{+}$. Again, using (5.2), we have

$$
\phi_{H_{1}}^{+}=\phi_{H_{2}}^{+}=\phi^{+}
$$

in $V_{R}^{+}$. Using 5.3, we get

$$
\log \left|c_{H_{1}}\right|^{d_{H_{2}}-1}=\log \left|c_{H_{2}}\right|^{d_{H_{1}}-1}+c\left(d_{H_{2}}-1\right)\left(d_{H_{1}}-1\right)
$$

which implies that

$$
c_{H_{1}}^{d_{H_{2}}} c_{H_{2}}=\delta_{+} c_{H_{2}}^{d_{H_{1}}} c_{H_{1}}
$$

where $\left|\delta_{+}\right|=e^{c\left(d_{H_{1}}-1\right)\left(d_{H_{2}}-1\right)}$.

By Proposition 5.1 ,

$$
\phi^{+} \circ H_{2} \circ H_{1}(x, y)=c_{H_{2}}\left(\phi^{+} \circ H_{1}(x, y)\right)^{d_{H_{2}}}=c_{H_{2}} c_{H_{1}}^{d_{H_{2}}}\left(\phi^{+}(x, y)\right)^{d_{H_{1}} d_{H_{2}}}
$$

and similarly,

$$
\phi^{+} \circ H_{1} \circ H_{2}(x, y)=c_{H_{1}}\left(\phi^{+} \circ H_{2}(x, y)\right)^{d_{H_{1}}}=c_{H_{1}} c_{H_{2}}^{d_{H_{1}}}\left(\phi^{+}(x, y)\right)^{d_{H_{1}} d_{H_{2}}} .
$$

Thus,

$$
\phi^{+}\left(H_{2} \circ H_{1}\right)=\delta_{+} \phi^{+}\left(H_{1} \circ H_{2}\right)
$$

on $V_{R}^{+}$. Now since

$$
\phi^{+} \circ H_{2} \circ H_{1}(x, y) \sim\left(H_{2} \circ H_{1}\right)_{2}(x, y)
$$

and

$$
\phi^{+} \circ H_{1} \circ H_{2}(x, y) \sim\left(H_{1} \circ H_{2}\right)_{2}(x, y)
$$

as $\|(x, y)\| \rightarrow \infty$ in $V_{R}^{+}$, it follows that for any fix $x_{0} \in \mathbb{C}$,

$$
\left(H_{2} \circ H_{1}\right)_{2}\left(x_{0}, y\right)-\delta_{+}\left(H_{1} \circ H_{2}\right)_{2}\left(x_{0}, y\right) \sim 0 \text { as }|y| \rightarrow \infty .
$$

Since the expression on the left of the above equation is a polynomial in $y$, it follows that

$$
\left(H_{2} \circ H_{1}\right)_{2}\left(x_{0}, y\right)=\delta_{+}\left(H_{1} \circ H_{2}\right)_{2}\left(x_{0}, y\right)
$$

for all $y \in \mathbb{C}$. Therefore,

$$
\left(H_{2} \circ H_{1}\right)_{2} \equiv \delta_{+}\left(H_{1} \circ H_{2}\right)_{2}
$$


in $\mathbb{C}^{2}$.

We again use Böttcher coordinates to recover the relation between the first components of these maps. As in the previous case, similarly one can show that

$$
\phi_{H_{1}}^{-}=\phi_{H_{2}}^{-}=\phi^{-} \text {. }
$$

Thus using Prop. 5.1, we get that

$$
\left(c_{H_{1}}^{\prime}\right)^{d_{H_{2}}} c_{H_{2}}^{\prime}\left(\phi^{-} \circ H_{1} \circ H_{2}(x, y)\right)^{d_{H_{1}} d_{H_{2}}}=\phi^{-}(x, y)
$$

and

$$
\left(c_{H_{2}}^{\prime}\right)^{d_{H_{1}}} c_{H_{1}}^{\prime}\left(\phi^{-} \circ H_{2} \circ H_{1}(x, y)\right)^{d_{H_{1}} d_{H_{2}}}=\phi^{-}(x, y)
$$

for all $(x, y) \in\left(H_{1} \circ H_{2}\right)^{-1}\left(V_{R}^{-}\right) \cap\left(H_{2} \circ H_{1}\right)^{-1}\left(V_{R}^{-}\right)=\mathcal{A}$. Note that $\mathcal{A}$ is an open neighbourhood of $I^{+}=[1: 0: 0]$ in $\mathbb{P}^{2}$. As in (5.9), it can be shown that

$$
\left(c_{H_{1}}^{\prime}\right)^{d_{H_{2}}} c_{H_{2}}^{\prime}=\eta\left(c_{H_{2}}^{\prime}\right)^{d_{H_{1}}} c_{H_{1}}^{\prime}
$$

where $|\eta|=e^{d\left(d_{H_{1}}-1\right)\left(d_{H_{2}}-1\right)}$ with $d=d_{1}-d_{2}$. Hence

$$
\left(\phi^{-} \circ H_{1} \circ H_{2}(x, y)\right)^{d_{H_{1}} d_{H_{2}}}=\eta\left(\phi^{-} \circ H_{2} \circ H_{1}(x, y)\right)^{d_{H_{1}} d_{H_{2}}}
$$

on $\mathcal{A}$. Consequently, there exists $\delta_{-}$(an appropriate $d_{H_{1}} d_{H_{2}}$-th root of $\eta$ ) such that

$$
\phi^{-} \circ\left(H_{1} \circ H_{2}\right)=\delta_{-} \phi^{-} \circ\left(H_{2} \circ H_{1}\right)
$$

on $\mathcal{A}$ where $\left|\delta_{-}\right|=e^{d\left(d_{H_{1}}-1\right)\left(d_{H_{2}}-1\right)}$.

Note that for a fixed $c \neq 0$, there exists $\epsilon>0$ sufficiently small such that

$$
\mathcal{A}_{\epsilon, c}=\{[1 / y: c: 1] \text { where } 0 \neq|y|<\epsilon\}
$$

intersects $\mathcal{A}$ and contains $I^{+}=[1: 0: 0]$ in its boundary. Choose $\left[x_{n}: c: 1\right] \in \mathcal{A}_{\epsilon, c}$ such that $\left|x_{n}\right| \rightarrow \infty$. Now since $\left(H_{2} \circ H_{1}\right)\left(x_{n}, c\right),\left(H_{1} \circ H_{2}\right)\left(x_{n}, c\right) \in V_{R}^{-}$, it follows that

$$
\left(H_{2} \circ H_{1}\right)_{1}\left(x_{n}, c\right),\left(H_{1} \circ H_{2}\right)_{1}\left(x_{n}, c\right) \rightarrow \infty
$$

as $n \rightarrow \infty$.

Using (5.10),

$$
\left(H_{1} \circ H_{2}\right)_{1}\left(x_{n}, c\right)-\delta_{-}\left(H_{2} \circ H_{1}\right)_{1}\left(x_{n}, c\right) \rightarrow 0
$$

as $n \rightarrow \infty$. The expression on the left is a polynomial in $x$ for each fixed $c$ and thus

$$
\left(H_{1} \circ H_{2}\right)_{1}(x, c)=\delta_{-}\left(H_{2} \circ H_{1}\right)_{1}(x, c)
$$

for all $x \in \mathbb{C}$. Using the similar argument as in the previous case, we get

$$
\left(H_{2} \circ H_{1}\right)_{1} \equiv \delta_{-}\left(H_{1} \circ H_{2}\right)_{1}
$$

in $\mathbb{C}^{2}$.

Hence using (5.6) and (5.11), we get

$$
H_{2} \circ H_{1}=C \circ H_{1} \circ H_{2}
$$

where $C(x, y)=\left(\delta_{-} x, \delta_{+} y\right)$ with $\left|\delta_{+}\right|=e^{c\left(d_{H_{1}}-1\right)\left(d_{H_{2}}-1\right)}$ and $\left|\delta_{-}\right|=e^{d\left(d_{H_{1}}-1\right)\left(d_{H_{2}}-1\right)}$ with $c=$ $c_{1}-c_{2}$ and $d=d_{1}-d_{2}$. 


\section{REFERENCES}

[1] Baker, I. N.; Erëmenko, A.: A problem on Julia sets. Ann. Acad. Sci. Fenn. Ser. A I Math. 12 (1987), no. 2, 229-236.

[2] Beardon, A. F.: Symmetries of Julia sets. Bull. London Math. Soc. 22 (1990), no. 6, 576-582.

[3] Beardon, A. F.: Polynomials with identical Julia sets. Complex Variables Theory Appl. 17 (1992), no. 3-4, 195-200.

[4] Bedford, E.; Smillie, J.: Polynomial diffeomorphisms of $\mathbb{C}^{2}$ : currents, equilibrium measure and hyperbolicity. Invent. Math. 103 (1991), no. 1, 69-99.

[5] Bedford, E.; Smillie, J.: Polynomial diffeomorphisms of $\mathbb{C}^{2}-I I$ : Stable manifolds and recurrence, J. Amer. Math. Soc. 4 (1991), pp. 657-679.

[6] Bedford, E.; Smillie, J.: Polynomial diffeomorphisms of $\mathbb{C}^{2}-$ III: Ergodicity, exponents and entropy of the equilibrium measure, Math. Ann. 294 (1992), pp. 395-420.

[7] Bera, S.; Pal, R.; Verma, K., A rigidity theorem for Hénon maps, European Journal of Mathematics (2019), https://doi.org/10.1007/s40879-019-00326-7

[8] Bisi, C.: On commuting polynomial automorphisms of $\mathbb{C}^{2}$. Publ. Mat. 48 (2004), no. 1, 227-239.

[9] Buzzard, G.; Formaess E.: Compositional roots of Hénon maps. Geometric complex analysis (Hayama, 1995), 67-73, World Sci. Publ., River Edge, NJ, 1996.

[10] Dinh, T.-C.; Sibony, N.: Rigidity of Julia sets for Hnon type maps. J. Mod. Dyn. 8 (2014), no. 3-4, 499-548.

[11] Fornæss, J. E.: Short $\mathbb{C}^{k}$. Complex analysis in several variablesMemorial Conference of Kiyoshi Oka's Centennial Birthday, 95108, Adv. Stud. Pure Math., 42, Math. Soc. Japan, Tokyo, 2004.

[12] Friedland, S.; Milnor, J.: Dynamical properties of plane polynomial automorphisms. Ergodic Theory Dynam. Systems 9 (1989), no. 1, 67-99.

[13] Jung, H. W. E.: Über ganze birationale transformationen der Ebene. J. Reine Angew. Math. 184(1942), $161-174$.

[14] Lamy, S.: L'alternative de Tits pour Aut $\mathbb{C}^{2}$. (French) [The Tits alternative for Aut( $\left.\mathbb{C}^{2}\right)$ ] J. Algebra 239 (2001), no. 2, 413-437.

[15] Levin, G.; Przytycki, F.: When do two rational functions have the same Julia set? (English summary) Proc. Amer. Math. Soc. 125 (1997), no. 7, 2179-2190.

[16] Morosawa, S., Nishimura, Y.; Taniguchi, M.; Ueda, T.: Holomorphic dynamics, Translated from the 1995 Japanese original and revised by the authors. Cambridge Studies in Advanced Mathematics, 66, Cambridge University Press, Cambridge, (2000).

[17] Schmidt, W.; Steinmetz, N.: The polynomials associated with a Julia set. (English summary) Bull. London Math. Soc. 27 (1995), no. 3, 239-241.

[18] Shafikov, R.; Wolf, C.: Filtrations, hyperbolicity, and dimension for polynomial automorphisms of $\mathbb{C}^{n}$. Michigan Math. J. 51 (2003), no. 3, 631-649.

[19] Sibony, N.: Dynamique des applications rationnelles de $\mathbb{P}^{k}$ in: Dynamique et géométrie complexes (Lyon, 1997), Panor. Synthéses, Soc. Math. France, Paris, 1999, pp. 97-185.

RP: Department of Mathematics, Indian Institute of Science Education and Research, Pune, MAHARASHTRA-411008, INDIA

E-mail address: ratna.math@gmail.com 